\title{
Conscious sedation guidance
}

\section{Conscious Sedation in Dentistry - Dental Clinical Guidance.}

Dundee: Scottish Dental Clinical Effectiveness Programme; 2006

Scope and purpose This guidance is intended to promote good clinical practice for the provision in dentistry of conscious sedation that is both safe and effective. It is not a recipe book for sedation and therefore does not include details of drug dosages. The recommendations are applicable to all patients receiving conscious sedation, to facilitate the provision of any type of dental treatment whether it is delivered in a dental practice, a community dental service clinic or a hospital setting. It also covers the provision of conscious sedation for dental treatment provided on a domiciliary basis. Specifically excluded from this guidance, however, are patients who require assisted ventilation, intensive care sedation, premedication for general anaesthesia, postoperative analgesia, sedation in palliative care, night sedation and sedation in the home setting other than for the provision of dental treatment on a domiciliary basis.

Methods Existing guidelines, relevant systematic reviews, policy documents, legislation or other recommendations were reviewed and appraised for their quality of development, evidence base and applicability to the remit of the guidance under development. To supplement this information, key questions were formulated by the Guidance Development Group and used as the basis for designing systematic literature search strategies to identify further research evidence that may address these questions, including unpublished work where relevant.

The following internet sites were searched for guidelines: New Zealand Guidelines Group, Canadian Collaboration on Clinical Practice Guidelines in Dentistry, National Guidelines Clearinghouse, FDI World Dental Federation, National Electronic Library for Health Guideline Finder, and Medline. The Cochrane Library was searched for systematic reviews and Medline, Embase and the Cochrane Library for studies to address key questions. The searches were supplemented by material already known to members of the Guidance Development Group.

Titles and abstracts of the identified references were screened for relevance independently by two researchers who were not members of the Guidance Development Group. Disagreement about the inclusion of specific individual references for further consideration was resolved by discussion and if necessary the opinion of a third researcher was sought. Included references were appraised and data was abstracted independently by two researchers using a specifically designed data-abstraction form. This information was then checked for inconsistencies, which were resolved by discussion, and used to construct evidence tables. The evidence tables were presented to the Guidance Development Group to inform their decision-making and their recommendations related to the key question under consideration.

Address for correspondence: Dr D Stirling, Scottish Dental Clinical Effectiveness Programme, Dundee Dental Education Centre, Frankland Building, Small's Wynd, Dundee, Scotland, DD1 4HN, UK.
Levels of evidence were assigned by two researchers who were not members of the Guidance Development Group. Formulation of each recommendation was achieved by consensus reached through discussion, drawing on the broad range of interest and experience of sedation related to dentistry within the membership of the Guidance Development Group.

Consultation and peer review were conducted prior to publication. A draft of the guidance was the subject of discussion at the Dental Sedation Teachers Group annual symposium in April 2004. Subsequently, approximately 100 copies were distributed throughout the UK to a range of professional organisations and individuals who have an interest in dental sedation, and comments were requested. In addition, all dentists in Scotland who recently claimed the National Health Service allowance for treatment with sedation were invited to comment. The consultation draft was also made available on the group's website (www.scottishdental.org/cep). All comments received through this consultation were considered and the guidance was amended accordingly prior to peer review. Further amendments were made in response to feedback from peer reviewers before publication.

Review and updating The guidance will be reviewed in 2 years' time (2008) and if there have been significant changes it will be updated accordingly.

Recommendations The detailed guidance make 48 recommendations in a range of areas:

\section{Referral}

- Discuss alternative methods of anxiety management with patient and ensure that dental care with sedation meets agreed definition of conscious sedation

\section{Assessment and record keeping}

- As part of a thorough assessment, discuss with patient all aspects of their conscious sedation treatment and also provide written instructions

- Obtain patient's written consent; maintain comprehensive and contemporaneous patient records

\section{Environment and facilities}

- Ensure that environment for sedation is safe and that correct equipment and drugs are provided for each sedation technique used

- Ensure that equipment and drugs for dealing with medical emergencies or complications related to sedation are immediately available

\section{Training}

- Ensure all members of dental team are correctly trained in sedation techniques used, including monitoring of patient during treatment and management of any sedation-related complications

- For oral and transmucosal sedation, ensure that sedationist is trained in other titratable techniques and skilled in performing venous cannulation

- Ensure that teams giving conscious sedation provide treatment for patient groups they are experienced in managing 


\section{Conscious sedation techniques}

- For inhalation sedation, ensure that a titrated dose of nitrous oxide is administered using dedicated purpose-designed equipment

- Oral, transmucosal and intravenous sedation require pulse oximetry and blood-pressure monitoring

- A titrated dose of midazolam is recommended for intravenous sedation

\section{Aftercare}

- Monitor patients throughout the recovery period until discharge by sedationist into the care of responsible adult escort who has also been given written postoperative instructions. An escort might not be required after nitrous oxide inhalation sedation

\section{Research recommendations}

A number of recommendations were made regarding the future conduct and reporting of clinical trials. The following areas were highlighted as requiring further high-quality research:

- Fasting before conscious sedation

- Conscious sedation of paediatric dental patients

- Dental conscious sedation using combinations of drugs

- Dental conscious sedation using continuous infusion

- The choice of sedation method for dental patients

- Cognitive and behavioural effects of conscious sedation

- The interaction of pharmacological and nonpharmacological anxiety management techniques.

The complete guidance is available for download at www.scottishdental.org/cep/guidance/dentalsedation.htm.

\section{Commentary}

This guidance has been produced for the dental team to help improve patient care, by bringing together the best available relevant information in an easily interpreted form for implementation. The guidance is for all patients receiving conscious sedation to facilitate dental treatment, in a primary care or hospital setting.

Members of the dental team develop a way of doing things, a routine, for managing patients with sedatives based on what they were taught, their own experiences and also perhaps influenced by respected or politically prominent individuals. Along the way one hopes that the routines are also modified by good research evidence.

The Scottish Dental Clinical Effectiveness Programme makes clear that they are presenting clinical "guidance" rather than "guidelines". The authors explain that this is because there is often a lack of high-quality evidence that would be necessary to inform conventional guidelines. They bring together legislation, policies and guidelines that are not yet readily accessible. They also include systematic reviews, but whether individual clinical trials are also included is unclear. The group does use methods similar to those used to develop high-quality guidelines, ie, they appraise the quality and evidence base of included items.

If this guidance is to be useful, it should detail specific clinical scenarios and offer specific recommendations for clinical management based on good evidence. It should also describe the nature of the evidence and it should be valid. Does this guidance do what it is supposed to do and help provide improved quality of patient care?

Several databases were searched although it is not clear whether there was a language restriction: clinical practice routines vary around the world. Presumably, although not stated, there were inclusion and exclusion criteria for the items to be brought together in this guidance because the product appears to describe UK current practice. The remit nevertheless seems to have been to produce Scottish rather than global guidance.

The guidance is comprehensive and describes preparation for sedation, conscious sedation techniques, conscious sedation for children, conscious sedation for adults and children with special needs, aftercare, records and documentation, clinical governance and training. The guidance concludes with recommendations for future research. Each section has clear statements with assigned levels of evidence and references where appropriate.

Most evidence is level 4 (expert opinion), but this is consistent with seeking to bring together policy documents and legislation. The guidance describes the state of current practice of sedation for dentistry in Scotland and is a useful document in doing this. It is also useful in that it informs the dental team about decision making and the quality of evidence supporting clinical practice. As most evidence is weak, however, the impact on clinical practice to be made by good research evidence is inevitably limited. More likely is that the guidance will provide a summary descriptive document of current practice that will reassure rather than stimulate improvement in the quality of patient care.

The guidance will be very useful for teaching and training. Even when systematic reviews in dentistry from the past are available it has been show that the quality could be improved. ${ }^{1}$ If future clinical decisions are to be based upon guidance then it is imperative that higher levels of evidence are available to inform them. This guidance is helpful in identifying key areas requiring further high quality research and in particular, randomised controlled trials.

\section{Paul Coulthard}

Unit of Oral and Maxillofacial Surgery, School of Dentistry, University of Manchester, Manchester, UK

1. Glenny AM, Esposito M, Coulthard P, Worthington HV. The assessment of systematic reviews in dentistry. Eur J Oral Sci 2003; 111:85-92.

Evidence-Based Dentistry (2006) 7, 81-82. doi:10.1038/sj.ebd.6400441 